\title{
Decreased duration of intravenous cephalosporins in intensive care unit patients with selective digestive decontamination: a retrospective before-and-after study
}

\author{
Calypso Mathieu $^{1} \cdot$ Roberta Abbate $^{1,2} \cdot$ Zoe Meresse $^{1} \cdot$ Emmanuelle Hammad $^{1} \cdot$ Gary Duclos $^{1} \cdot$ François Antonini $^{1}$. \\ Nadim Cassir ${ }^{3}$. Jeroen Schouten ${ }^{4} \cdot$ Laurent Zieleskiewicz $^{1} \cdot$ Marc Leone $^{1,3,5}$ (1)
}

Received: 11 February 2020 / Accepted: 25 June 2020 / Published online: 2 July 2020

(C) Springer-Verlag GmbH Germany, part of Springer Nature 2020

\begin{abstract}
Selective digestive decontamination (SDD) reduces the rate of infection and improves the outcomes of patients admitted to an intensive care unit (ICU). A risk associated with its use is the development of multi-drug-resistant organisms. We hypothesized that a 1-day reduction in systemic antimicrobial exposure in the SDD regimen would not affect the outcomes of our patients. In this before-and-after study design, 199 patients and 248 patients were included in a 3-day SDD group and a 2-day SDD group, respectively. The rates of hospital-acquired pneumonia and ICU infections were similar in both groups. The rates of bloodstream infection and bacteriuria were significantly lower in the 2-day SDD group than in the 3-day SDD group. Compared with the patients in the 3-day group, the patients in the 2-day SDD group received fewer antibiotics and less exposure to mechanical ventilation, and they used fewer ICU resources. The rates of ICU mortality and 28-day mortality were similar in both groups. The incidence of multi-drug-resistant organisms was similar in both groups. Within the limitations inherent to our study design, reducing the exposure of prophylactic systemic antibiotics in the SDD setting from 3 days to 2 days was not associated with impaired outcomes. Future randomized controlled trials should be conducted to test this hypothesis and investigate the effects on the development of multi-drug resistant organisms.
\end{abstract}

Keywords Infection $\cdot$ Selective $\cdot$ Decontamination $\cdot$ Prophylaxis $\cdot$ Antibiotic

Electronic supplementary material The online version of this article (https://doi.org/10.1007/s10096-020-03966-w) contains supplementary material, which is available to authorized users.

Marc Leone

marc.leone@ap-hm.fr

1 Aix Marseille University, Assistance Publique Hôpitaux de Marseille, Hôpital Nord, Service d'anesthésie et de réanimation, Marseille, France

2 Department of Anesthesiology, University of Naples Federico II, Naples, Italy

3 MEPHI, IHU Méditerranée Infection, Aix-Marseille Université, Marseille, France

4 Department of Intensive Care, Radboudumc, Netherlands

5 Chemin des Bourrely, Service d'anesthésie et de réanimation, 13015 Marseille, France

\section{Introduction}

Infection is a major cause of morbidity and mortality in intensive care units (ICU) [1]. The most common ICU-acquired infections are due to hospital-associated pneumonia (HAP), which were reported to be 15 to 18 episodes per 1000 ventilator days in Europe and 12\% in French healthcare Networks [2, 3].

In selective digestive decontamination (SDD), an enteral and oropharyngeal paste containing antimicrobials is combined with short-term systemic prophylactic antibiotics. In ICU patients, SDD has been associated with reductions in hospital mortality, HAP, and the duration of mechanical ventilation [4-8]. The French guidelines for HAP suggest the routine use of SDD to prevent HAP in units with low levels of antibiotic resistance [9]. Historically, the duration of systemic prophylactic antibiotic administration has been 4 days, but this duration has ranged from 2 to 5 days in previous studies [10].

Prolonged antibiotic therapy may lead to the emergence of multi-drug-resistant (MDR) organisms [11, 12]. Because the 
major fear associated with SDD use is the development of MDR organisms, reduction in the exposure to systemic antibiotics could decrease this risk, but it could be associated with the decreased efficiency of the procedure.

We hypothesized that the reduction in the duration of the systemic prophylactic antibiotic would not affect the patient outcomes. In this before-and-after study, we aimed to evaluate the effects on the occurrence of HAP of the decrease in the duration of a systemic prophylactic antibiotic in the SDD setting from 3 to 2 days. The secondary goals were to assess the rates of ICU-acquired infections, the number of antibiotic-free days, ventilator-free days, vasopressor-free days, and ICUfree days, in addition to the septic shock and ICU- and 28day mortality rates in the 2-day and 3-day study periods.

\section{Materials and methods}

\section{Study design and patients}

From February 2014 to March 2018, we conducted a retrospective before-and-after study in the 15-bed ICU of a 625 bed university hospital (Hôpital Nord, Marseille, France). All patients admitted to the ICU for trauma, coma, or cardiac arrest with a length of stay of at least 3 days and requiring tracheal intubation at admission were included. Patients admitted for infection and receiving curative antibiotics or patients with inappropriate treatment (incomplete, duration error) were not included. The SDD regimen was started on the day of tracheal intubation.

The oropharyngeal paste contained polymyxin E (2\%), tobramycin (2\%), and amphotericin B (2\%). Enteral mixture was administered through a nasogastric tube in $10 \mathrm{ml}$ of a suspension containing 100-mg polymyxin E, 80-mg tobramycin, and 500-mg amphotericin B. The application was performed three times daily until tracheal extubation in all patients. The systemic prophylactic antibiotic was cefazolin, which was administered intravenously at $1 \mathrm{~g}$ every $8 \mathrm{~h}$, except in patients reporting an allergy (no systematic antibiotic in these patients). The patients were managed according to international guidelines [9]. In our ICU, antimicrobial stewardship is based on the protocols described elsewhere [13, 14].

In February 2016, the duration of systemic prophylactic antibiotic administration was reduced from 3 to 2 days to decrease the patients' exposure to the antibiotic. The patients admitted from February 1, 2014, to February 1, 2016, were included in the 3-day SDD group, and those admitted from March 1, 2016, to March 1, 2018, were included in the 2-day SDD group.

\section{Data collection}

Demographic and clinical features, including age, gender, simplified acute physiology score (SAPS II), and the reason for admission were collected from our electronic database. We recorded HAP and other ICU-acquired infections, including catheter-associated urinary tract infection (CAUTI), bloodstream infection, and others (e.g., meningitis and intraabdominal infection) between the day of admission and day 28. Once a week, all diagnosis of infection are discussed and reviewed by the same group of senior intensivists and an infectious diseases physician, and decisions are based according to international definitions (Supplemental data 1). The same definition was used during the pre- and post-intervention periods. We also recorded bacterial and Candida spp. colonization in the lung and urine, MDR organism infection and/or colonization, candidemia, and Clostridium difficile infection. We recorded the number of patients who received narrow-spectrum $\beta$ lactams, which were then defined by the use of cloxacillin, amoxicillin, amoxicillin-clavulanate, and cephalosporin; broad-spectrum $\beta$-lactams defined by use of piperacillin-tazobactam, ceftazidime, cefepime, carbapenem; and other antibiotics defined by use of fluoroquinolone, gentamicin, amikacin, vancomycin, linezolid, and metronidazole. The number of antibiotic-free days (excluding the antibiotics used in SDD), ventilator-free days, vasopressor-free days, and ICU-free days were reported from the day of admission to day 28 or the day of death, as applicable. Septic shock was defined according to the international definition [15]. We recorded the ICU readmission rates, ICU rates, and 28-day mortality rates. Patients with missing data were excluded from the analysis.

\section{Statistical analysis}

The statistical analyses were performed using the R-Project 3.4.4 software for Ubuntu Linux. The quantitative data were expressed in means with standard deviation. The quantitative data were compared using an analysis of variance ANOVA. A Student's t test was used when the data distribution was normal. A Kruskal-Wallis (or Mann-Whitney) test was used if it was not normal. The qualitative data were expressed as absolute numbers and percentages. Proportion comparisons were made using a chi-squared test or an exact Fisher test. A value of $p<0.05$ was used as the final significance threshold. A multivariate logistic regression analysis was performed. The relevant variables of $p<0.2$ in the univariate analysis were included in a stepwise variable selection model. A value of $p<0.05$ was used as the final significance threshold. Our study was based on a before-and-after design and included all patients who met the inclusion criteria during the two study periods. However, to ensure a power of $90 \%$ with an alpha value of $5 \%$ and a $12 \%$ rate of hospital-associated pneumonia [3], the sample size required per group was 93 patients.

\section{Ethical considerations}

In this observational retrospective study, based on the French legislation on biomedical research (articles L. 1121-1 
paragraph 1 and R. 1121-3, Public Health Code), no ethical problems were present, which was confirmed by the Comité d'Ethique pour la Recherche en Anesthésie-Réanimation (CERAR) [16]. The patients and their families were informed that their data could be used in this study during their ICU stay. The data were stored and treated according to the Commission Nationale de l'Informatique et des Libertés (CNIL) authorization number 2018-32.

\section{Results}

During the study period, 3233 patients were admitted to our ICU. Of these patients, 1471 were admitted between February 1, 2014, and February 1, 2016, and 1762 were admitted between March 1, 2016, and March 1, 2018, (Fig. 1). Among these patients, 505 were admitted for trauma, coma, or cardiac arrest with mechanical ventilation and an ICU length of stay of 3 days or more. Fifty-eight patients were not included because they required antibiotic therapy at admission for a (suspected) infection or inappropriate treatment (absence or incomplete). Finally, 199 and 248 patients were included in the 3-day SDD group and the 2-day SDD group, respectively.

The results of the univariate analysis are shown in Table 1. The demographic data were similar in both groups. Although the number of HAPs was similar in both groups, the rate of bloodstream infections was lower in the 2-day SDD group than in the 3-day SDD group (10 [4\%] and 21 [11\%], respectively; $p=0.012$ ). The number of MDR organisms collected in the urine, lungs, and infected sites was similar in both groups (10 [5\%] and 10 [4\%], respectively, $p=0.784)$. Candida colonization and C. difficile infections did not differ between the two groups (Table 1). The number of bacteriuria was significantly lower in the 2-day SDD group than in the 3day SDD group (14 [6\%)] and 28 [14\%], respectively; $p=$ 0.004).

In the 2-day SDD group, the numbers of antibiotic-free days, mechanical ventilator-free days, and ICU-free days were higher than in the 3-day SDD group. The ICU-mortality and 28-day mortality rates did not differ significantly in the two groups.

The multivariate analysis showed that the 3-day SDD group was an independent factor associated with the increased risk of bacteriuria and bloodstream infection, whereas it was a protective factor associated with the number of ventilator-free days (Table 2).

\section{Discussion}

In our study, the reduced duration of the administration of a systemic prophylactic antibiotic from 3 to 2 days was not

Fig. 1 Flow chart

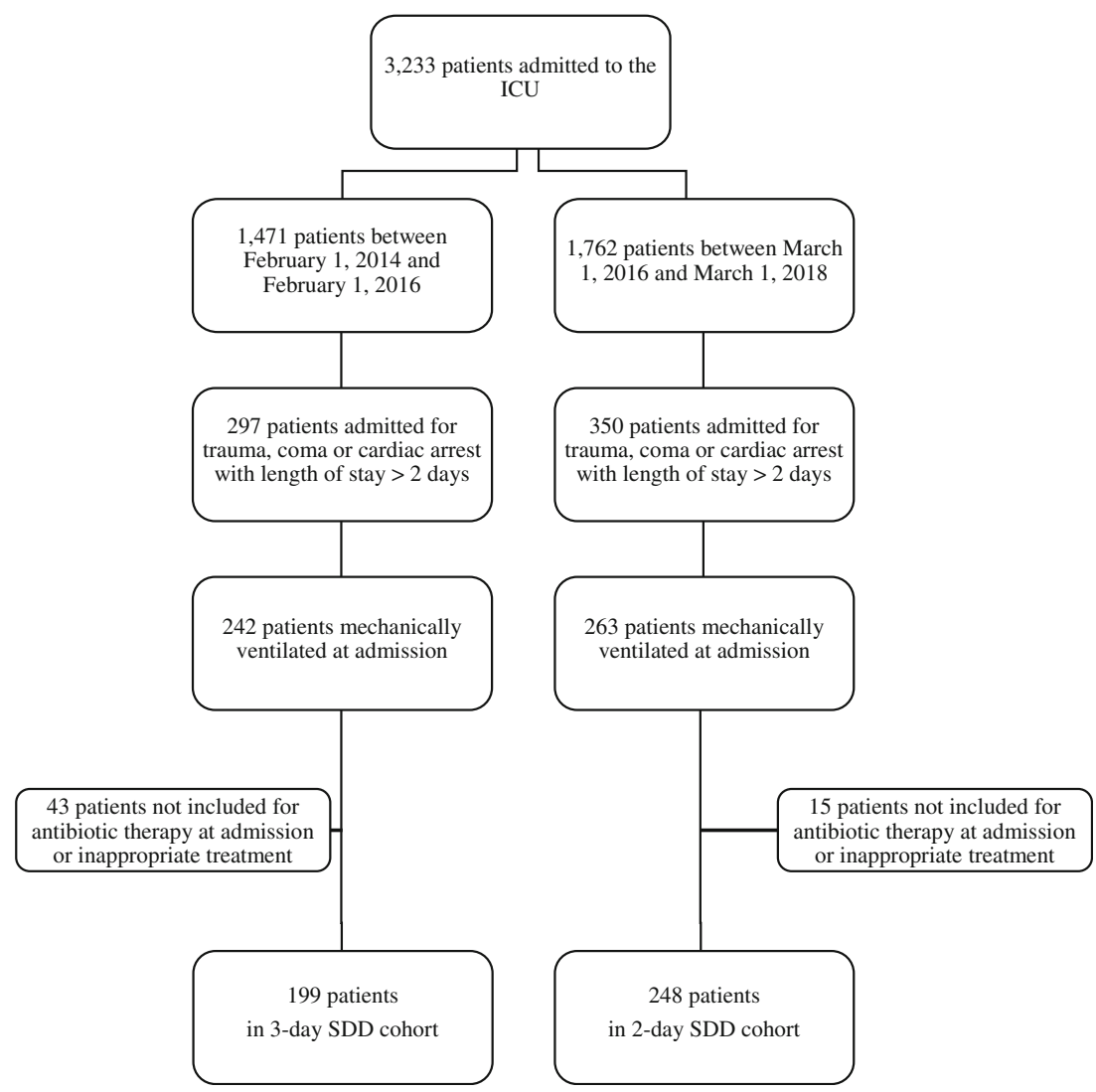


Table 1 Results of univariate analysis

\begin{tabular}{|c|c|c|c|}
\hline Variable & 3-day $\operatorname{SDD}(n=199)$ & 2-day SDD $(n=248)$ & $P$ value \\
\hline Age (yr.), mean \pm SD & $47 \pm 20$ & $48 \pm 21$ & 0.947 \\
\hline Sex, male no $(\%)$ & $146(73)$ & $197(79)$ & 0.163 \\
\hline SAPS II score, mean \pm SD & $53 \pm 15$ & $53 \pm 16$ & 0.538 \\
\hline Reason for admission, no (\%) & & & 0.160 \\
\hline Trauma & $148(74)$ & $200(81)$ & \\
\hline Coma & $35(18)$ & $28(11)$ & \\
\hline Cardiac arrest & $16(8)$ & $20(8)$ & \\
\hline ICU infection, no (\%) & $47(24)$ & $58(23)$ & 1.000 \\
\hline HAP & $23(12)$ & $28(11)$ & 1.000 \\
\hline CAUTI & $9(5)$ & $15(6)$ & 0.617 \\
\hline Bloodstream infection & $21(11)$ & $10(4)$ & 0.012 \\
\hline Others & $12(6)$ & $13(5)$ & 0.878 \\
\hline Bacterial colonization, no $(\%)$ & $55(28)$ & $54(22)$ & 0.185 \\
\hline Lung colonization & $32(16)$ & $43(17)$ & 0.821 \\
\hline Bacteriuria & $28(14)$ & $14(6)$ & 0.004 \\
\hline Candida colonization, no (\%) & $18(9)$ & $15(6)$ & 0.307 \\
\hline MDR bacteria, no (\%) & $10(5)$ & $10(4)$ & 0.784 \\
\hline Candidemia, no (\%) & $0(0)$ & $1(0.4)$ & 1.000 \\
\hline Clostridium difficile infection, no (\%) & $1(0.5)$ & $1(0.4)$ & 1.000 \\
\hline Antibiotic-free days (d), mean \pm SD & $19 \pm 10$ & $21 \pm 9$ & 0.036 \\
\hline \multicolumn{4}{|l|}{ Antibiotics administered, No (\%) } \\
\hline Narrow-spectrum $\beta$-lactams & $27(14)$ & $35(14)$ & 0.978 \\
\hline Broad-spectrum $\beta$-lactams & $41(21)$ & $44(18)$ & 0.519 \\
\hline Others & $31(16)$ & $38(15)$ & 1.000 \\
\hline Septic shock, no (\%) & $11(6)$ & $8(3)$ & 0.336 \\
\hline Ventilator-free days (d), mean $\pm \mathrm{SD}$ & $12 \pm 11$ & $15 \pm 11$ & $<0.001$ \\
\hline Vasopressor-free days (d), mean $\pm \mathrm{SD}$ & $19 \pm 10$ & $20 \pm 10$ & 0.534 \\
\hline ICU-free days $(\mathrm{d})$, mean $\pm \mathrm{SD}$ & $10 \pm 10$ & $12 \pm 10$ & 0.011 \\
\hline ICU readmission, no (\%) & $12(6)$ & $12(5)$ & 0.731 \\
\hline ICU mortality, no (\%) & $63(32)$ & $63(25)$ & 0.175 \\
\hline 28-day mortality, no (\%) & $63(32)$ & $66(27)$ & 0.287 \\
\hline
\end{tabular}

SAPS II, simplified acute physiology score; ICU, intensive care unit; HAP, hospital-acquired pneumonia; CAUTI, catheter-associated urinary tract infection; $M D R$, multi-drug resistant associated with an increase in HAP, ICU infections, or 28-day mortality rate. In comparison, the administration of the

Table 2 Results of multivariate logistic regression analysis of 3-day selective digestive decontamination

\begin{tabular}{lll}
\hline Variable & OR & $95 \% \mathrm{CI}$ \\
\hline Bacteriuria & 2.54 & $1.28-5.27$ \\
Bloodstream infection & 2.84 & $1.28-6.67$ \\
Ventilator-free days & 0.94 & $0.88-0.99$ \\
ICU-free days & 1.05 & $0.99-1.12$ \\
\hline
\end{tabular}

$O R$, odd ratio; $C I$, confidence interval; $I C U$, intensive care unit. The relevant variables of $p<0.2$ in the univariate analysis were included in a stepwise variable selection model systemic prophylactic antibiotic to the patients in the 3-day group was associated with higher rates of bacteriuria and bloodstream infections, increased exposure to mechanical ventilation, and increased exposure to antibiotics.

In the SDD setting, topical antimicrobials alone decreased the rate of respiratory infection, but this strategy did not affect the mortality rates [5]. An effect on mortality was found in patients treated using a combination of topical and systemic prophylactic antimicrobials $[5,6,17]$. In most previous studies, the duration of systemic treatment was 4 days. A fixed duration of 4 days comes from studies carried out in the 1980s. The rational was a systemic prophylaxis administration until most potentially pathogenic microorganisms colonizing the patient are eliminated by SDD $[10,18]$. Many studies compared short- versus long-term antibiotic prophylaxis in surgery 
without showing any difference on surgical site infections [19-21]. To our knowledge, this study is the first to investigate the duration of systemic antibiotics in SDD.

The use of SDD is controversial, particularly a systemic prophylactic antibiotic. The European guidelines for the management of HAP suggest the use of selective oral decontamination, but not SDD [22]. The main reason for the restriction on its use is the uncertain effect on bacterial resistance, especially in parts of the world where the prevalence of resistant microorganisms is higher [23]. However, SDD has been found to be effective and safe in environments with low levels of resistance [23-25]. In trauma patients, prophylactic antibiotic days were identified as an independent predictor of MDR organism development [12], where patients who developed MDR organism pneumonia received on average 7 days of prophylactic antibiotics, whereas those who developed susceptible HAP received only 2 days of prophylactic antibiotics [12]. In the present study, we modified our protocol by reducing the number of prophylactic antibiotic days. In our study, the prevalence of MDR organisms in the lung, urine, or blood of the patients in our sample was around 5\%. In our ICU, systematic rectal sampling for MDR organisms during the stay was implemented in 2018. Therefore, we were unable to compare the ecological effects over the two periods of the study. However, the analysis of other sites did not show significant differences in MDR organism colonization or infection; therefore, it could be hypothesized that decreased antimicrobial exposure would not have deleterious effects on the ecology.

Previous studies showed that SDD decreased the number of bloodstream infections [26, 27]. In our study, the decrease in the duration of the systemic prophylactic antibiotic was associated with decreased numbers of bloodstream infections, bacteriuria, exposure to antibiotics (excluding those used for SDD), and days without mechanical ventilation. It should be noted that variables not included in our analyses may have affected these findings. Although these results may also have been due to a change in practice between the two periods, they confirmed the efficacy of administering systemic prophylactic antibiotics for SDD during a short period.

Our study has several limitations. The retrospective design was based on before-and-after analyses. Hence, although our protocols and practices were similar during the two study periods, unrecognized changes may have affected our findings. However, the two study populations were similar. It is possible that other factors that could influence the results were not included such as co-morbidities and the use of catheters. However, the rates of catheter-associated bloodstream infections and catheter-associated urinary tract infections were stable during the study period (around 2\% and 5-6\% in 2016 and 2018 , respectively). Colonization was defined by isolation of a micro-organism without evidence of infection. As the samples were ordered by the judgment of a clinician, these results have to be interpreted with caution. In addition, because this study was conducted in a single ICU, further research is required to confirm the generalizability of the results to other ICUs. The ecology of our patients cannot be assumed to reflect that in other ICUs because only $5 \%$ of pathogens were considered MDR organisms. The French guidelines on HAP in ICUs were published in November 2017. Because our study period was from February 2014 to March 2018, the influence of these guidelines on our results was probably limited; moreover, SDD has been used in our ICU for several years [28-30]. Bundles of care for the prevention of HAP have also been widely used in our ICU [9]. As explained above, the systematic use of rectal sampling was reintroduced in 2018, which made it difficult to assess the rectal colonization of the patients in our study. Finally, to limit the potential for bias, possible confounding factors were included in the analysis.

In conclusion, although unmeasured confounding factors may account for our results, the decrease in the duration of systemic prophylactic antibiotic SDD from 3 to 2 days was not associated with an increase in ICU infections. So, this strategy seems safe while reducing the amount of systemic antibiotics used. This observation needs to be replicated in other ICUs. A prospective and multi-center randomized controlled trial should be conducted to evaluate the optimal duration of the administration of systemic prophylactic antibiotics as a component of the SDD regimen.

\section{Compliance with ethical standards}

Conflict of interest ML received fees from $3 \mathrm{M}$, Aguettant, Amomed, Aspen, BioMerieux, MSD, Octapharma, Orion, Pfizer.

Ethical approval In this observational retrospective study, based on the French legislation on biomedical research (articles L. 1121-1 paragraph 1 and R. 1121-3, Public Health Code), no ethical problems were present, which was confirmed by the Comité d'Ethique pour la Recherche en Anesthésie-Réanimation (CERAR) [16]. The patients and their families were informed that their data could be used in this study during their ICU stay. The data were stored and treated according to the Commission Nationale de l'Informatique et des Libertés (CNIL) authorization number 2018-32.

Informed consent The patients and their families were informed that their data could be used in this study during their ICU stay.

\section{References}

1. Vincent J-L, Rello J, Marshall J et al (2009) International study of the prevalence and outcomes of infection in intensive care units. JAMA 302:2323-2329. https://doi.org/10.1001/jama.2009.1754

2. Koulenti D, Tsigou E, Rello J (2017) Nosocomial pneumonia in 27 ICUs in Europe: perspectives from the EU-VAP/CAP study. Eur J Clin Microbiol Infect Dis 36:1999-2006. https://doi.org/10.1007/ s10096-016-2703-z

3. Savey A (2017) Surveillance des infections nosocomiales en réanimation adulte, Réseau REA-Raisin, France. Résultats. 82 
http://www.cpias-auvergnerhonealpes.fr/Reseaux/REA/Resultat/ resultat_nationaux/rapport national_rea_2017.pdf

4. Plantinga NL, de Smet AMGA, Oostdijk E a N et al (2018) Selective digestive and oropharyngeal decontamination in medical and surgical ICU patients: individual patient data meta-analysis. Clin Microbiol Infect 24:505-513. https://doi.org/10.1016/j.cmi. 2017.08.019

5. Liberati A, D'Amico R, Pifferi S et al (2009) Antibiotic prophylaxis to reduce respiratory tract infections and mortality in adults receiving intensive care Cochrane Database Syst Rev:CD000022. https:// doi.org/10.1002/14651858.CD000022.pub3

6. Roquilly A, Marret E, Abraham E, Asehnoune K (2015) Pneumonia prevention to decrease mortality in intensive care unit: a systematic review and meta-analysis. Clin Infect Dis 60:64-75. https://doi.org/10.1093/cid/ciu740

7. de Smet AMGA, JW KJ a, Cooper BS et al (2009) Decontamination of the digestive tract and oropharynx in ICU patients. N Engl J Med 360:20-31. https://doi.org/10.1056/ NEJMoa0800394

8. Daneman N, Sarwar S, Fowler RA et al (2013) Effect of selective decontamination on antimicrobial resistance in intensive care units: a systematic review and meta-analysis. Lancet Infect Dis 13:328341. https://doi.org/10.1016/S1473-3099(12)70322-5

9. Leone M, Bouadma L, Bouhemad B et al (2018) Hospital-acquired pneumonia in ICU. Anaesth Crit Care Pain Med 37:83-98. https:// doi.org/10.1016/j.accpm.2017.11.006

10. Stoutenbeek CP, van Saene HKF, Miranda DR, Zandstra DF (1984) The effect of selective decontamination of the digestive tract on colonisation and infection rate in multiple trauma patients. Intensive Care Med 10:185-192. https://doi.org/10.1007/ BF00259435

11. Roquilly A, Feuillet F, Seguin P et al (2016) Empiric antimicrobial therapy for ventilator-associated pneumonia after brain injury. Eur Respir J 47:1219-1228. https://doi.org/10.1183/13993003.013142015

12. Lewis RH, Sharpe JP, Swanson JM et al (2018) Reinventing the wheel: impact of prolonged antibiotic exposure on multidrugresistant ventilator-associated pneumonia in trauma patients. J Trauma Acute Care Surg 85:256-262. https://doi.org/10.1097/TA. 0000000000001936

13. Leone M, Roberts JA, Bassetti M et al (2019) Update in antibiotic therapy in intensive care unit: report from the 2019 Nîmes International Symposium. Anaesth Crit Care Pain Med 38:647656. https://doi.org/10.1016/j.accpm.2019.09.009

14. Mathieu C, Pastene B, Cassir N et al (2019) Efficacy and safety of antimicrobial de-escalation as a clinical strategy. Expert Rev AntiInfect Ther 17:79-88. https://doi.org/10.1080/14787210.2019. 1561275

15. Singer M, Deutschman CS, Seymour CW et al (2016) The third international consensus definitions for sepsis and septic shock (Sepsis-3). JAMA 315:801-810. https://doi.org/10.1001/jama. 2016.0287

16. Toulouse E, Masseguin C, Lafont B et al (2018) French legal approach to clinical research. Anaesth Crit Care Pain Med 37:607614. https://doi.org/10.1016/j.accpm.2018.10.013

17. Price R, MacLennan G, Glen J, SuDDICU Collaboration (2014) Selective digestive or oropharyngeal decontamination and topical oropharyngeal chlorhexidine for prevention of death in general intensive care: systematic review and network meta-analysis. BMJ 348:g2197. https://doi.org/10.1136/bmj.g2197

18. Stoutenbeek CP, van Saene HKF (1990) Infection prevention in intensive care by selective decontamination of the digestive tract.
J Crit Care 5:137-156. https://doi.org/10.1016/0883-9441(90) 90058-H

19. Lador A, Nasir H, Mansur $\mathrm{N}$ et al (2012) Antibiotic prophylaxis in cardiac surgery: systematic review and meta-analysis. J Antimicrob Chemother 67:541-550. https://doi.org/10.1093/jac/dkr470

20. Oxman DA, Issa NC, Marty FM et al (2013) Postoperative antibacterial prophylaxis for the prevention of infectious complications associated with tube thoracostomy in patients undergoing elective general thoracic surgery: a double-blind, placebo-controlled, randomized trial. JAMA Surg 148:440-446. https://doi.org/10.1001/ jamasurg.2013.1372

21. Wang F, Chen X-Z, Liu J et al (2012) Short-term versus long-term administration of single prophylactic antibiotic in elective gastric tumor surgery. Hepatogastroenterology 59:1784-1788. https://doi. org/10.5754/hge11784

22. Torres A, Niederman MS, Chastre J et al (2017) International ERS/ ESICM/ESCMID/ALAT guidelines for the management of hospital-acquired pneumonia and ventilator-associated pneumonia: Guidelines for the management of hospital-acquired pneumonia (HAP)/ventilator-associated pneumonia (VAP) of the European Respiratory Society (ERS), European Society of Intensive Care Medicine (ESICM), European Society of Clinical Microbiology and Infectious Diseases (ESCMID) and Asociación Latinoamericana del Tórax (ALAT). Eur Respir J 50. https://doi. org $/ 10.1183 / 13993003.00582-2017$

23. Cavalcanti AB, Lisboa T, Gales AC (2017) Is selective digestive decontamination useful for critically ill patients? Shock 47:52-57. https://doi.org/10.1097/SHK.0000000000000711

24. Buitinck S, Jansen R, Rijkenberg S et al (2019) The ecological effects of selective decontamination of the digestive tract (SDD) on antimicrobial resistance: a 21-year longitudinal single-centre study. Crit Care 23:208. https://doi.org/10.1186/s13054-0192480-z

25. Wittekamp BHJ, Oostdijk EAN, Cuthbertson BH et al (2019) Selective decontamination of the digestive tract (SDD) in critically ill patients: a narrative review. Intensive Care Med. https://doi.org/ 10.1007/s00134-019-05883-9

26. Sánchez-Ramírez C, Hípola-Escalada S, Cabrera-Santana M et al (2018) Long-term use of selective digestive decontamination in an ICU highly endemic for bacterial resistance. Crit Care 22:141. https://doi.org/10.1186/s13054-018-2057-2

27. Silvestri L, van Saene HKF, Milanese M et al (2007) Selective decontamination of the digestive tract reduces bacterial bloodstream infection and mortality in critically ill patients. Systematic review of randomized, controlled trials. J Hosp Infect 65:187-203. https://doi.org/10.1016/j.jhin.2006.10.014

28. Quinio B, Albanèse J, Bues-Charbit M et al (1996) Selective decontamination of the digestive tract in multiple trauma patients. A prospective double-blind, randomized, placebo-controlled study. Chest 109:765-772. https://doi.org/10.1378/chest.109.3.765

29. Leone M, Albanese J, Antonini F et al (2003) Long-term (6-year) effect of selective digestive decontamination on antimicrobial resistance in intensive care, multiple-trauma patients. Crit Care Med 31: 2090-2095. https://doi.org/10.1097/01.CCM.0000079606.16776. C5

30. Leone M, Delliaux S, Bourgoin A et al (2005) Risk factors for lateonset ventilator-associated pneumonia in trauma patients receiving selective digestive decontamination. Intensive Care Med 31:64-70. https://doi.org/10.1007/s00134-004-2514-z

Publisher's note Springer Nature remains neutral with regard to jurisdictional claims in published maps and institutional affiliations. 